\title{
Efficacy and Safety of Tofacitinib Re-treatment for Ulcerative Colitis After Treatment Interruption: Results from the OCTAVE Clinical Trials
}

\author{
Julian Panés, ${ }^{\mathrm{a}, \mathrm{e}}$ Séverine Vermeire, ${ }^{\mathrm{b}}$ Marla C. Dubinsky, \\ Edward V. Loftus Jr, ${ }^{d}$ Nervin Lawendy, ${ }^{e}$ Wenjin Wang, ${ }^{e}$ Leonardo Salese, \\ Chinyu Su, ${ }^{\mathrm{e}}$ Irene Modesto, ${ }^{\mathrm{f}}$ Xiang Guo, ${ }^{\mathrm{e}}$ Jean-Frederic Colombel ${ }^{\mathrm{g}, \ominus}$
}

\begin{abstract}
alnflammatory Bowel Disease Unit, Hospital Clínic de Barcelona, IDIBAPS, CIBERehd, Barcelona, Spain Department of Gastroenterology, University Hospitals Leuven, Leuven, Belgium 'Department of Pediatrics, Icahn School of Medicine at Mount Sinai, New York, NY, USA dDivision of Gastroenterology and Hepatology, Mayo Clinic College of Medicine, Rochester, MN, USA ePfizer Inc, Collegeville, PA, USA fPfizer Inc, New York, NY, USA 'Division of Gastroenterology, Icahn School of Medicine at Mount Sinai Hospital, New York, NY, USA
\end{abstract}

Corresponding author: Dr Julian Panés, MD, Inflammatory Bowel Disease Unit, Hospital Clínic de Barcelona, IDIBAPS, CIBERehd, Barcelona, Spain. Tel.: +34-93-2275418; email: jpanes@clinic.cat

\begin{abstract}
Background and Aims: Tofacitinib is an oral, small molecule Janus kinase inhibitor for the treatment of ulcerative colitis. Here, we evaluate the efficacy and safety of tofacitinib re-treatment following treatment interruption in patients with ulcerative colitis.

Methods: Here, patients with clinical response to tofacitinib $10 \mathrm{mg}$ b.d. induction therapy were randomised to receive placebo in OCTAVE Sustain. Those experiencing treatment failure after Week 8 of OCTAVE Sustain entered OCTAVE Open and re-initiated tofacitinib $10 \mathrm{mg}$ b.d. [re-treatment subpopulation]; efficacy and safety data are presented up to Month 36 of OCTAVE Open.

Results: Median time to treatment failure following interruption was 169 (95\% confidence interval $[\mathrm{Cl}], 94.0-179.0)$ and 123 [95\% Cl, 91.0-168.0] days for induction remitters, and induction responders but non-remitters, respectively. Following re-treatment with tofacitinib, rates (nonresponder imputation after a patient discontinued; latest observation carried forward imputation after a patient advanced to a subsequent study [NRI-LOCF]) of clinical response, remission, and endoscopic improvement were $74.0 \%, 39.0 \%$, and $55.0 \%$ at Month 2 , and $48.5 \%, 37.4 \%$, and $42.4 \%$ at Month 36, respectively. Among induction remitters and induction responders but non-remitters, clinical response rates at Month 36 were $60.6 \%$ and $42.4 \%$ [NRI-LOCF], respectively. Efficacy was recaptured regardless of prior tumour necrosis factor inhibitor failure status. The safety profile of tofacitinib $10 \mathrm{mg}$ b.d. re-treatment was consistent with the overall cohort and demonstrated no new safety risks associated with exposure of $\leq 36$ months.

Conclusions: Median time to treatment failure was numerically higher in induction remitters versus induction responders but non-remitters. Following treatment interruption, efficacy was safely and successfully recaptured with tofacitinib $10 \mathrm{mg}$ b.d. re-treatment in a substantial proportion of patients [ClinicalTrials.gov:NCT01458574;NCT01470612].
\end{abstract}

Key Words: Re-treatment; tofacitinib; ulcerative colitis

(C) The Author(s) 2021. Published by Oxford University Press on behalf of European Crohn's and Colitis Organisation. This is an Open Access article distributed under the terms of the Creative Commons Attribution-NonCommercial License 


\section{Introduction}

Ulcerative colitis [UC] is a chronic, idiopathic disease of the colon characterised by inflammation and a relapsing and remitting course. ${ }^{1}$ The aim of therapy is to achieve symptomatic, endoscopic, and histological remission. ${ }^{2}$

For patients with UC, pharmacological therapy may be interrupted or stopped to accommodate a number of scenarios, including pregnancy, surgery, illness, infection, comorbidities, adverse events, or a change in patient's insurance. ${ }^{3}$ It is therefore important for physicians managing patients with UC to understand the possible clinical consequences of temporarily discontinuing a therapy. Considerations include the rapidity of drug clearance following discontinuation, the median time to relapse, and subsequent expectations around the recapture of efficacy following re-treatment. ${ }^{3}$

Many patients with inflammatory bowel disease are primary non-responders, or exhibit secondary loss of response to biologic therapies such as tumour necrosis factor inhibitors [TNFi]. ${ }^{4}$ One contributor to this loss of response is immunogenicity; biologics are large proteins and can stimulate the production of neutralising anti-drug antibodies, which can not only reduce the efficacy of the biologic, but can also potentially induce adverse events. ${ }^{4,5}$ Whereas reported rates of anti-drug antibody formation in patients with inflammatory bowel disease treated with biologics are highly variable and can be influenced by various factors, including prior exposure to biologics, a meta-analysis assessing the immunogenicity of biologic therapies revealed that up to $65.3 \%$ of patients with inflammatory bowel disease treated with infliximab were reported to have developed antidrug antibodies. ${ }^{4}$ Furthermore, although concomitant treatment with immunomodulator therapy is associated with reduced magnitude of immunogenic response to $\mathrm{TNFi}^{6}{ }^{6}$ this may carry an additional risk of infection ${ }^{7}$ and cancer. ${ }^{8,9}$ Therefore, as neutralising anti-drug antibody formation has been reported to contribute to secondary loss of response to biologics, it may also limit the efficacy and safety of re-treatment with biologics following temporary interruption. ${ }^{3}$

Tofacitinib is an oral, small molecule Janus kinase inhibitor for the treatment of UC. The efficacy and safety of tofacitinib have been evaluated in an 8 -week, phase 2 induction study, ${ }^{10}$ three phase 3, randomised, placebo-controlled studies [OCTAVE Induction 1, OCTAVE Induction 2, and OCTAVE Sustain $],{ }^{11}$ and an open-label, long-term extension [OLE] study [OCTAVE Open] ${ }^{12}$ in patients with moderately-to-severely active UC. Pharmacodynamic effects can persist for up to 6 weeks. ${ }^{13}$ As tofacitinib is a small molecule, it is not expected to elicit the formation of neutralising anti-drug antibodies that may limit successful re-treatment. ${ }^{14,15}$

In this study, we evaluated efficacy outcomes in patients who had clinical response to tofacitinib (10 or $15 \mathrm{mg}$ twice daily [b.d.]) induction therapy followed by a period of treatment interruption of up to 52 weeks. Efficacy and safety were subsequently evaluated in patients requiring re-initiation of tofacitinib $10 \mathrm{mg}$ b.d. following treatment failure while receiving placebo. Furthermore, we evaluated efficacy outcomes stratified by prior TNFi failure and remission status at baseline of OCTAVE Sustain.

\section{Materials and Methods}

\subsection{Study design}

The full details of OCTAVE Induction 1 and 2 [NCT01465763 and NCT01458951], OCTAVE Sustain [NCT01458574], and OCTAVE Open [NCT01470612] have been reported previously. ${ }^{11,12}$ The present analysis included patients who achieved clinical response at the end of the 8-week OCTAVE Induction 1 and 2 studies with tofacitinib 10 or $15 \mathrm{mg}$ b.d. and entered the OCTAVE Sustain maintenance study receiving placebo. Clinical response was defined as a decrease from induction study baseline total Mayo score of $\geq 3$ points and $\geq 30 \%$, plus a decrease in rectal bleeding subscore of $\geq 1$ point or an absolute rectal bleeding subscore of 0 or 1 . Patients experiencing treatment failure between Week 8 and Week 52 of OCTAVE Sustain were eligible to enrol into OCTAVE Open to receive tofacitinib $10 \mathrm{mg}$ b.d. Treatment failure was defined as an increase from maintenance study baseline total Mayo score of $\geq 3$ points, plus an increase in rectal bleeding subscore and endoscopic subscore of $\geq 1$ point, and an absolute endoscopic subscore $\geq 2$ points, after $\geq 8$ weeks of maintenance therapy.

Oral corticosteroids [prednisone-equivalent up to $25 \mathrm{mg} / \mathrm{day}$ ] were permitted during the induction studies, provided that the dose remained stable for at least 2 weeks prior to baseline and throughout the induction study period. Corticosteroid tapering was mandatory at the beginning of OCTAVE Sustain and OCTAVE Open, although doses $<10 \mathrm{mg} /$ day were permitted in OCTAVE Open. Prohibited concomitant therapies included TNFi and immunomodulators.

\subsection{Patients}

These post hoc analyses evaluated two subgroups of patients: the treatment interruption subpopulation and the re-treatment subpopulation.

\subsubsection{Treatment interruption subpopulation}

The treatment interruption subpopulation consisted of patients who achieved clinical response following 8 weeks of induction therapy with tofacitinib [10 or $15 \mathrm{mg}$ b.d.] and were randomised to receive placebo during OCTAVE Sustain [Figure 1].

\subsubsection{Re-treatment subpopulation}

The re-treatment subpopulation consisted of patients from the 'treatment interruption' subpopulation who received tofacitinib $10 \mathrm{mg}$ b.d., experienced treatment failure between Week 8 and Week 52 of OCTAVE Sustain [while receiving placebo], and subsequently entered OCTAVE Open and received tofacitinib $10 \mathrm{mg}$ b.d. [Figure 1].

\subsection{Efficacy measures}

Clinical response, remission, and endoscopic improvement endpoints were assessed in the overall treatment interruption subpopulation at Weeks 24 and 52 of OCTAVE Sustain, based on centrally read endoscopic subscore [Figure 1]. Remission was defined as a total Mayo score of $\leq 2$ with no individual subscore $>1$, and a rectal bleeding subscore of 0 . Endoscopic improvement [defined as mucosal healing in the original OCTAVE protocols ${ }^{11,12]}$ was defined as a Mayo endoscopic subscore of 0 or 1 . Patients with missing binary efficacy values were treated as non-responders for the corresponding binary efficacy outcomes. Median time to treatment failure, in days, was estimated for the treatment interruption subpopulation, while proportions of patients with treatment failure were estimated from the KaplanMeier curves for the time-to-treatment-failure endpoint. This efficacy outcome was stratified by the remission status at baseline of OCTAVE Sustain. Induction remitters were defined as patients with a total Mayo score of $\leq 2$ and no individual subscore $>1$, and a rectal bleeding subscore of 0 at baseline of OCTAVE Sustain. Induction responders but non-remitters were defined as patients who had clinical response but were not in remission at baseline of OCTAVE Sustain.

In the re-treatment subpopulation, the endpoints of clinical response, remission, and endoscopic improvement were assessed at Months 2, 12, 24, and 36 of OCTAVE Open after re-initiation of 


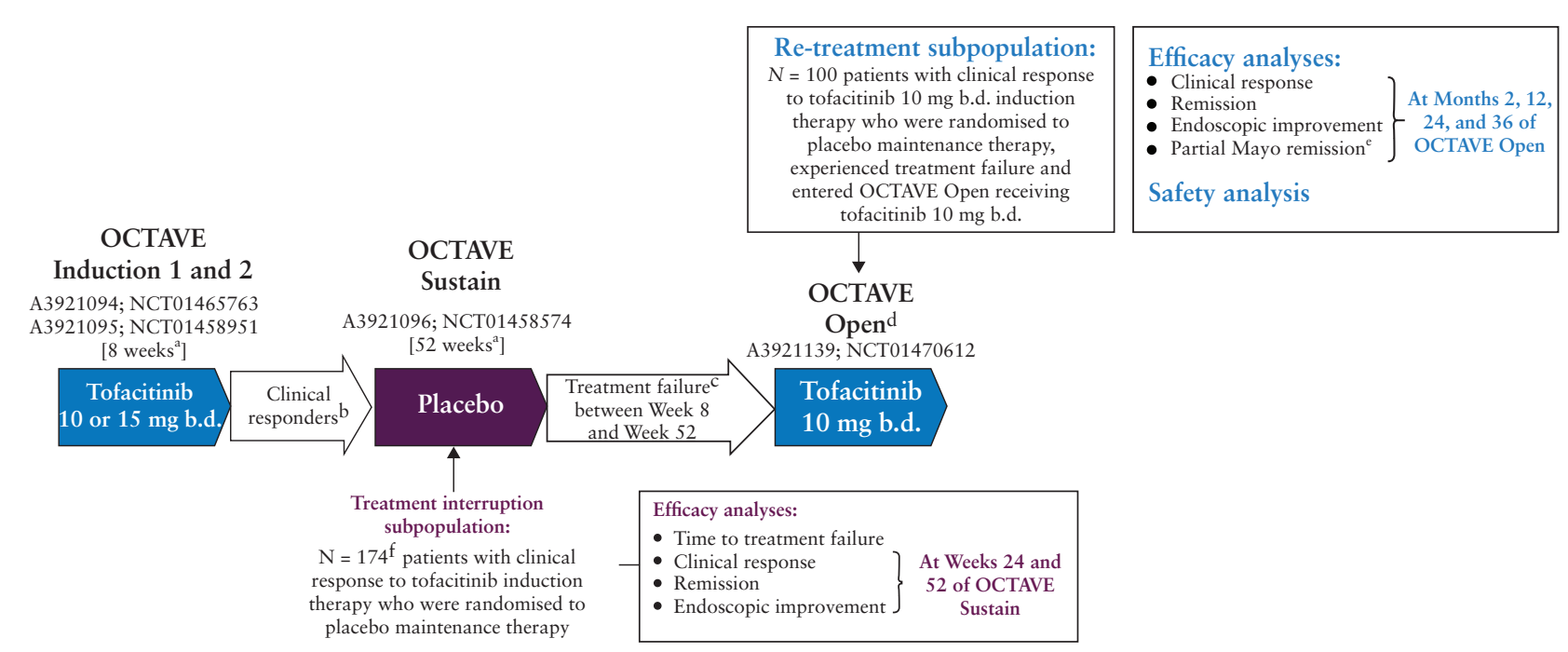

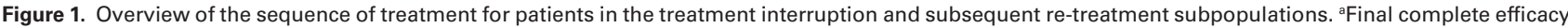
assessment at Week 8/52. Treatment continued up to Week 9/53. ${ }^{b} \mathrm{Clinical}$ response in OCTAVE Induction 1 and 2 was defined as a decrease from induction study baseline total Mayo score of $\geq 3$ points and $\geq 30 \%$, plus a decrease in rectal bleeding subscore of $\geq 1$ point or an absolute rectal bleeding subscore of 0 or 1. ${ }^{c}$ Treatment failure was defined as an increase from maintenance study baseline total Mayo score of $\geq 3$ points, plus an increase in rectal bleeding subscore and endoscopic subscore of $\geq 1$ point, and an absolute endoscopic subscore $\geq 2$ points, after $\geq 8$ weeks of maintenance therapy. ${ }^{\mathrm{d} S t u d y}$ A3921139 [OCTAVE Open] was ongoing at the time of this interim analysis. ePartial Mayo remission was assessed at Months 1, 9, 21, and 33. ${ }^{\mathrm{T}}$ Two patients without clinical response were randomised into OCTAVE Sustain [protocol deviations]. b.d., twice daily; $N$, number of evaluable patients.

tofacitinib $10 \mathrm{mg}$ b.d., based on locally read endoscopic subscore [Figure 1]. Efficacy data from OCTAVE Open are presented up to May 27, 2019 [database not locked]. Furthermore, partial Mayo score, which encompasses stool frequency, rectal bleeding, and Physician's Global Assessment of disease activity subscores, was assessed from Month 1 of OCTAVE Open, with partial Mayo remission defined as a partial Mayo score of $\leq 2$ with no individual subscore $>1$. These efficacy outcomes were further analysed by prior TNFi failure status and by remission status at baseline of OCTAVE Sustain. For re-treatment subpopulation efficacy outcomes, both observed and non-responder imputation [NRI] latest observation carried forward [LOCF] data are reported. NRI was applied after a patient discontinued, and LOCF was applied after a patient advanced to a subsequent study up to the visit they would have reached if they had stayed in the study. Patients without clinical response at Month 2 discontinued from the study and were considered non-responders for all endpoints at Months 12, 24 , and 36 . No imputation was applied for ongoing patients, except NRI for intermittent missing data.

Stepwise logistic regression analyses were performed to evaluate the association of baseline variables [a full list of the variables included are detailed in the Supplementary Information, available as Supplementary data at ECCO-JCC online] with the recapture of clinical response at Months 2, 12, 24, and 36 following tofacitinib re-treatment. Odds ratios [ORs] and $95 \%$ confidence intervals [CIs] were reported. Nominal $p$-values of $\leq 0.05$ were considered significant.

\subsection{Safety assessments}

Safety was assessed in all patients in the re-treatment subpopulation who received $\geq 1$ dose of tofacitinib $10 \mathrm{mg}$ b.d. in OCTAVE Open (up to May 27, 2019 [database not locked]), with no imputation for missing data. Safety data for the induction and maintenance studies have been described previously. ${ }^{11}$ Safety data in the overall cohort of the tofacitinib UC clinical programme [all patients receiving tofacitinib 5 or $10 \mathrm{mg}$ b.d. in phase 3 or OLE studies] are presented for contextualisation. ${ }^{16}$
Proportions and incidence rates [IRs; unique patients with events per 100 patient-years of exposure], with $95 \%$ CIs for safety events of special interest, were calculated using an exact method. Opportunistic infections, malignancies [including non-melanoma skin cancer], and cardiovascular events [including major adverse cardiovascular events] were reviewed by independent adjudication committees.

\subsection{Ethical consideration}

All studies were registered with ClinicalTrials.gov [NCT01458574; NCT01470612] and were conducted in compliance with the Declaration of Helsinki and the International Conference on Harmonization Good Clinical Practice Guidelines, and were approved by the Institutional Review Boards and/or Independent Ethics Committees at each investigational centre participating in the studies or at a central Institutional Review Board. All patients provided written informed consent.

\section{Results}

\subsection{Efficacy following treatment interruption}

\subsubsection{Patients - Treatment interruption subpopulation}

This analysis included 174 patients who received tofacitinib induction therapy [10 or $15 \mathrm{mg}$ b.d.] and were randomised to receive placebo in OCTAVE Sustain. Following 8 weeks of induction therapy, 172 patients achieved clinical response, and two patients without clinical response were randomised into OCTAVE Sustain as protocol deviations. The majority of these patients had prior immunosuppressant [67.8\%] or corticosteroid [74.7\%] failure. Overall, $47.1 \%$ of patients had prior TNFi failure [Supplementary Table 1, available as Supplementary data at ECCO-JCC online]. Furthermore, seven patients received tofacitinib $15 \mathrm{mg}$ b.d. in OCTAVE Induction 1 and 2 , a dose that was subsequently discontinued following a protocol amendment. These seven patients, in addition to the two patients without clinical response [total of nine patients], were not included in the subsequent tofacitinib $10 \mathrm{mg}$ b.d. induction responder treatment 
interruption subpopulation comprising induction remitters [ $n=49]$ and induction responders but non-remitters $[n=116]$ subgroups.

Baseline demographics and disease characteristics of the treatment interruption subpopulation [overall and stratified by remission status] are presented in Supplementary Table 1.

\subsubsection{Efficacy responses following treatment interruption}

At baseline of OCTAVE Sustain, 98.9\% [172/174] of patients in the overall treatment interruption subpopulation had a clinical response following tofacitinib induction therapy. Following treatment interruption with placebo, the proportion of patients with clinical response declined to $32.2 \%$ [56/174] and $19.0 \%$ [33/174] at Weeks 24 and 52 of OCTAVE Sustain, respectively [Figure 2A]. Similar trends were observed for the proportion of patients with efficacy outcomes of remission (29.9\% [52/174], 9.8\% [17/174], and 10.3\% [18/174]) and endoscopic improvement $(50.0 \%$ [87/174], 15.5\% [27/174], and $12.6 \%$ [22/174]) at baseline, Week 24, and Week 52 of OCTAVE Sustain, respectively [Figure 2A].

\subsubsection{Time to treatment failure following treatment} interruption

Within the overall treatment interruption subpopulation, the cumulative rate of treatment failure after 52 weeks of placebo treatment was $75.3 \%$ [95\% CI, 67.8-81.3], with a median time to treatment failure of 135 days [95\% CI, 95.0-168.0] after tofacitinib interruption [Supplementary Figure 1, available as Supplementary data at ECCO-JCC online].

Among the 49/165 [29.7\%] induction remitter patients, the median time to treatment failure was 169 days [95\% CI, 94.0-179.0]; the corresponding value for induction responders but non-remitters was 123 days [95\% CI, 91.0-168.0]. Cumulative rates of treatment failure for induction remitters, versus induction responders but non-remitters, receiving placebo were generally similar up to Week 52 of OCTAVE Sustain, as shown by overlapping 95\% CIs [Supplementary Table 2, available as Supplementary data at ECCO$J C C$ online]. This was also true when the rates were further stratified by prior TNFi failure status [Supplementary Table 2]. At Week 8 in OCTAVE Sustain, Kaplan-Meier rates of treatment failure were $21.7 \%$ [95\% CI, 11.2-34.5] in induction remitters versus $29.0 \%$ [95\% CI, 20.9-37.4] in induction responders but non-remitters [Figure 2B and Supplementary Table 2]. At Week 52, rates of treatment failure in induction remitters were $81.8 \%$ [95\% CI, 67.0-90.4] versus $72.4 \%$ [95\% CI, 62.7-80.0] in induction responders but nonremitters [Figure 2B and Supplementary Table 2].

\subsection{Efficacy and safety of tofacitinib re-treatment following treatment failure}

\subsubsection{Patients - Re-treatment subpopulation}

The re-treatment subpopulation of OCTAVE Open comprised 100 patients with clinical response to tofacitinib $10 \mathrm{mg}$ b.d. in OCTAVE Induction 1 and 2, and subsequent treatment failure with placebo in OCTAVE Sustain. These patients were enrolled in OCTAVE Open for at least 24 months prior to the data cut-off date. Baseline demographics and disease characteristics of the re-treatment subpopulation are presented in Table 1 .

\subsubsection{Efficacy following tofacitinib re-treatment}

3.2.2.1. Overall re-treatment subpopulation

The proportions of patients in the re-treatment subpopulation achieving clinical response, partial Mayo remission, remission, or endoscopic improvement during OCTAVE Open are shown in Figure 3. Clinical response was recaptured in $74.0 \%$ [95\% CI, 65.4-82.6] of patients at Month 2 of OCTAVE Open, and 48.5\% [95\% CI, 38.6-58.3] of patients at Month 36 [NRI-LOCF]. Corresponding observed data were $85.1 \%$ [95\% CI, 77.6-92.6] and 94.0\% [95\% CI, 87.4-100.0] of patients, respectively [Figure 3A].

Mean partial Mayo score showed that efficacy could be recaptured as early as Month 1 of tofacitinib re-treatment in OCTAVE Open; mean partial Mayo score decreased to 2.4 versus 6.6 at baseline of OCTAVE Open. Furthermore, mean partial Mayo score was 1.2 by Month 4 . In the overall re-treatment subpopulation, partial Mayo remission was achieved in 50.0\% [95\% CI, 40.2-59.8] of patients as early as Month 1, and in 52.0\% [95\% CI, 42.2-61.8] of patients at Month 33 [NRI]. Corresponding observed data were $54.3 \%$ [95\% CI, 44.2-64.5] and 96.3\% [95\% CI, 91.3-100.0] of patients at Months 1 and 33, respectively [Figure 3B].

Remission was achieved at Month 2 of tofacitinib re-treatment by $39.0 \%$ [95\% CI, 29.4-48.6] of patients [NRI]. At Months 12 and $36,43.0 \%$ [95\% CI, 33.3-52.7] [NRI] and 37.4\% [95\% CI, 27.8-46.9] [NRI-LOCF] of patients achieved remission, respectively. Corresponding observed data were $44.8 \%$ [95\% CI, 34.4-55.3], $61.4 \%$ [ $95 \%$ CI, 50.0-72.8], and $72.0 \%$ [95\% CI, 59.6-84.5] of patients, respectively [Figure $3 \mathrm{~A}$ ].

Endoscopic improvement was achieved at Month 2 of tofacitinib re-treatment by $55.0 \%$ [95\% CI, 45.3-64.8] of patients [NRI]. At Months 12 and 36, 54.0\% [95\% CI, 44.2-63.8] [NRI] and 42.4\% [95\% CI, 32.7-52.2] [NRI-LOCF] of patients achieved endoscopic improvement, respectively. Corresponding observed data were 61.1\% [95\% CI, 51.0-71.2], 75.0\% [95\% CI, 65.0-85.0], and $78.8 \%$ [95\% CI, 67.8-90.0] of patients, respectively [Figure 3A].

\subsubsection{Prior TNFi failure status}

Among patients with prior TNFi failure, clinical response was recaptured in $80.0 \%$ [95\% CI, 68.3-91.7], 66.7\% [95\% CI, 52.9-80.4], and $40.0 \%$ [95\% CI, 25.7-54.3] of patients at Months 2, 12, and 36, respectively [NRI] [Figure 4A]. Corresponding observed data were $92.3 \%$ [95\% CI, 83.9-100.0], 93.8\% [95\% CI, 85.4-100.0], and $89.5 \%$ [95\% CI, 75.7-100.0] of patients, respectively. The proportions of patients achieving clinical response, partial Mayo remission, remission, and endoscopic improvement efficacy endpoints up to Month 36 were unaffected by prior TNFi failure status [Figure $4 \mathrm{~A}$ and $\mathrm{B}]$.

\subsubsection{Remission status at baseline of OCTAVE Sustain}

The proportions of patients with a clinical response up to Month 36 were similar regardless of remission status at baseline of OCTAVE Sustain [NRI-LOCF and observed data; Figure 5A].

A greater proportion of induction remitters achieved partial Mayo remission at Month 1 versus induction responders but nonremitters [NRI and observed data; Figure 5B]. From Month 9 to Month 33, rates of partial Mayo remission were similar between induction remitters and induction responders but non-remitters [observed data]. At Month 33, 66.7\% [95\% CI, 50.6-82.8] of induction remitters and $44.8 \%$ [95\% CI, 32.9-56.7] of induction responders but non-remitters achieved partial Mayo remission [NRI; Figure 5B].

A greater proportion of induction remitters achieved remission or endoscopic improvement at Month 2 versus induction responders but non-remitters. By Month 24, the proportions of patients in remission or with endoscopic improvement were similar regardless of remission status [NRI-LOCF and observed data; Figure 5A]. 

A
Baseline of OCTAVE Sustain
Week 24 of OCTAVE Sustain
Week 52 of OCTAVE Sustain

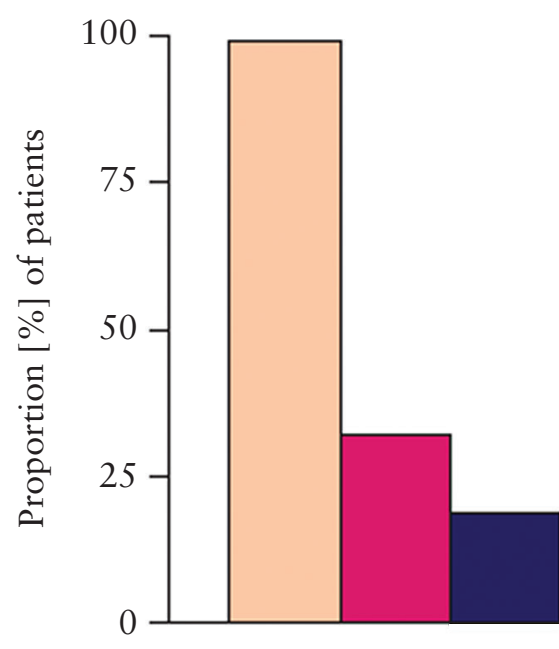

Clinical response

$\begin{array}{cccc}n & 172 & 56 & 33 \\ N^{\mathrm{a}} & 174 & 174 & 174\end{array}$

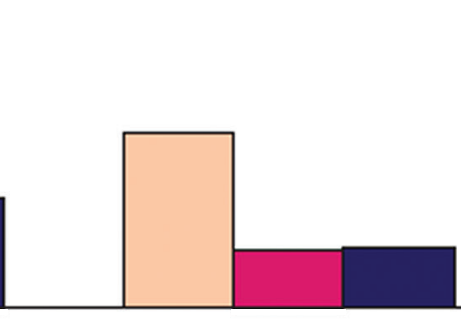

Remission

$\begin{array}{lll}52 & 17 & 18\end{array}$

$174 \quad 174 \quad 174$

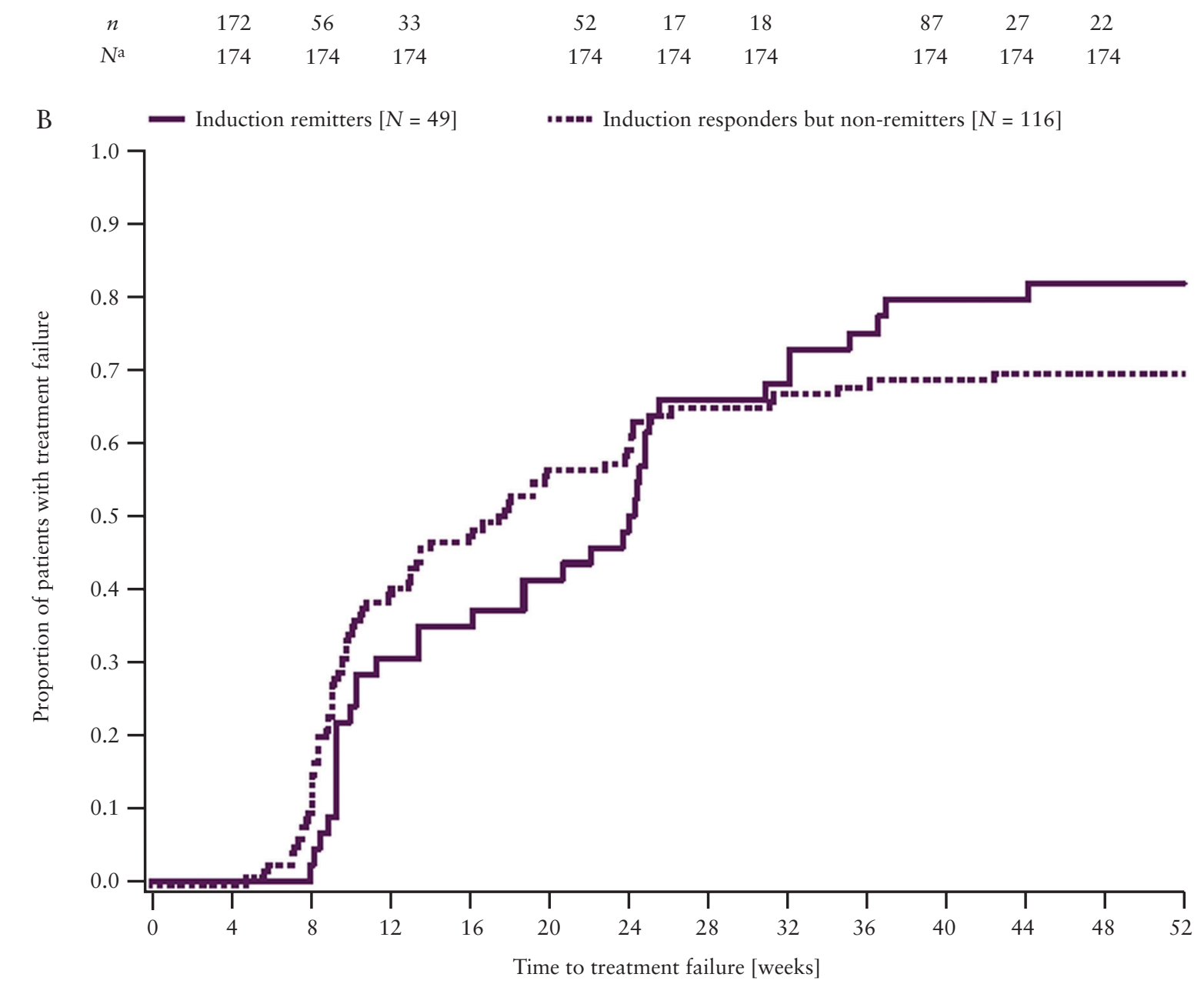

Figure 2. [A] Efficacy responses in the overall treatment interruption subpopulation at baseline and Weeks 24 and 52 of OCTAVE Sustain, and [B] Kaplan-Meier curves of time to treatment failure during OCTAVE Sustain in induction remitters and induction responders but non-remitters. The overall treatment interruption subpopulation comprised 174 patients who had 8 weeks of tofacitinib induction therapy [10 or 15 mg b.d.] and were randomised to placebo in OCTAVE Sustain. Clinical response was defined as a decrease from induction study baseline total Mayo score of $\geq 3$ points and $30 \%$, plus a decrease in rectal bleeding subscore of $\geq 1$ point or an absolute rectal bleeding subscore of 0 or 1 . Endoscopic improvement was defined as a Mayo endoscopic subscore of 0 or 1 . Remission was defined as a total Mayo score of $\leq 2$ with no individual subscore $>1$, and a rectal bleeding subscore of 0 . alncludes seven patients who received tofacitinib $15 \mathrm{mg}$ b.d. in OCTAVE Induction, and two patients without clinical response were randomised into OCTAVE Sustain [protocol deviations]. b.d., twice daily; $N$, number of patients treated in the treatment group; $n$, number of patients with efficacy response. 
Table 1. Demographics and clinical characteristics of the tofacitinib re-treatment subpopulation from baseline of OCTAVE Open.

\begin{tabular}{|c|c|c|c|c|c|}
\hline & \multirow[t]{2}{*}{ All patients $[N=100]$} & \multicolumn{2}{|c|}{ Prior TNFi failure } & \multicolumn{2}{|c|}{$\begin{array}{l}\text { Remission at baseline of } \\
\text { OCTAVE Sustain }\end{array}$} \\
\hline & & Yes $[N=45]$ & No $[N=55]$ & Yes $[N=33]$ & No $[N=67$ \\
\hline Age $[y]$, mean $[S D]^{a}$ & $44.2[13.7]$ & $47.8[13.4]$ & $41.4[13.4]$ & $46.2[12.7]$ & $43.3[14.2]$ \\
\hline Male, $n[\%]$ & $61[61.0]$ & $25[55.6]$ & $36[65.5]$ & $22[66.7]$ & $39[58.2]$ \\
\hline \multicolumn{6}{|l|}{ Race, $n[\%]$} \\
\hline White & $82[82.0]$ & 37 [82.2] & 45 [81.8] & $27[81.8]$ & $55[82.1]$ \\
\hline Black & $1[1.0]$ & $0[0.0]$ & $1[1.8]$ & $1[3.0]$ & $0[0.0]$ \\
\hline Asian & $10[10.0]$ & 5 [11.1] & $5[9.1]$ & $4[12.1]$ & $6[9.0]$ \\
\hline Other & $4[4.0]$ & $1[2.2]$ & $3[5.5]$ & $1[3.0]$ & $3[4.5]$ \\
\hline Unspecified & $3[3.0]$ & $2[4.4]$ & $1[1.8]$ & $0[0.0]$ & $3[4.5]$ \\
\hline Total Mayo score at baseline, mean [SD] & $9.2[1.6]$ & $9.1[1.7]$ & $9.3[1.6]$ & $8.3[1.8]$ & $9.7[1.4]$ \\
\hline Partial Mayo score at baseline, mean [SD] & $6.6[1.5]$ & $6.6[1.6]$ & $6.7[1.5]$ & $5.9[1.6]$ & $7.0[1.3]$ \\
\hline Prior TNFi failure, $n[\%]^{a}$ & $45[45.0]$ & $45[100.0]$ & $0[0.0]$ & $13[39.4]$ & $32[47.8]$ \\
\hline Prior immunosuppressant failure, $n[\%]^{\mathrm{a}}$ & $73[73.0]$ & $38[84.4]$ & $35[63.6]$ & $22[66.7]$ & $51[76.1]$ \\
\hline Prior corticosteroid failure, $n[\%]^{a}$ & $74[74.0]$ & $35[77.8]$ & $39[70.9]$ & $28[84.8]$ & $46[68.7]$ \\
\hline Corticosteroid use at baseline, $n[\%]$ & $12[12.0]$ & $6[13.3]$ & $6[10.9]$ & $3[9.1]$ & $9[13.4]$ \\
\hline Duration of disease, y $[S D]^{a}$ & $8.8[7.8]$ & $10.4[6.7]$ & $7.6[8.5]$ & $9.2[8.8]$ & $8.7[7.4]$ \\
\hline
\end{tabular}

The re-treatment subpopulation comprised patients who had clinical response at Week 8 with tofacitinib 10 mg b.d. in OCTAVE Induction 1 or 2, and subsequent treatment failure with placebo during OCTAVE Sustain. Per protocol, these patients received tofacitinib 10 mg b.d. in the OLE study; remission was defined as a total Mayo score of $\leq 2$ with no individual subscore $>1$, and a rectal bleeding subscore of 0 .

b.d., twice daily; $N$, number of patients in the re-treatment subpopulation; $n$, number of patients within the given category; OLE, open-label, long-term extension; SD, standard deviation; TNFi, tumour necrosis factor inhibitor; y, years.

${ }^{a}$ Data at baseline of induction studies.

\subsubsection{Predictors of recapture of efficacy}

In multivariable logistic regression modelling, age, total Mayo score at baseline of OCTAVE Open, prior immunosuppressant failure, and oral corticosteroid use at baseline of OCTAVE Induction were significant predictors of recapturing efficacy following re-treatment [Table 2]. Total Mayo score at baseline of OCTAVE Open was a potential predictor of recapture of response at Months 2 and 12 of tofacitinib re-treatment (OR [per 1-point increase] 0.61 [95\% CI, 0.40-0.91] and 0.69 [95\% CI, 0.51-0.93]), respectively [Table 2]. Patients with lower total Mayo score at baseline of OCTAVE Open were more likely to recapture clinical response at Months 2 and 12. Increasing age was associated with recapture of response at Months 2 and 36 of tofacitinib re-treatment (OR [per 10-year increment] 1.59 [95\% CI, 1.06-2.38] and 1.54 [95\% CI, 1.09-2.17]), respectively) and no prior immunosuppressant failure was significantly associated with recapture of response at Month 36 (OR 3.51 [95\% CI, 1.21-10.15]) [Table 2]. Patients with no oral corticosteroid use at baseline of OCTAVE Induction 1 and 2 were more likely to recapture response at Months 2, 24, and 36 of tofacitinib re-treatment (OR 5.37 [95\% CI, 1.72-16.72], 3.32 [95\% CI, 1.45-7.59], and 2.61 [95\% CI, 1.09-6.24]), respectively). Other potential predictors, including oral corticosteroid use at baseline of OCTAVE Open, prior TNFi failure, and remission status at baseline of OCTAVE Sustain were not significantly associated with the recapture of clinical response up to Month 36 of tofacitinib re-treatment in this multivariable analysis.

\subsubsection{Safety following re-treatment}

A summary of safety and events of special interest in the re-treatment subpopulation is presented in Table 3. Safety data in the overall cohort [all patients receiving tofacitinib 5 or $10 \mathrm{mg}$ b.d. in phase 3 or OLE studies] are presented for contextualisation. Serious adverse events occurred in $25.0 \%$ [25/100] of patients in the re-treatment subpopulation and $12.0 \%$ [12/100] of adverse events resulted in discontinuation up to Month 36 . The most frequently reported serious adverse event was serious infection [ $n=7]$, with an overall IR of 2.7 [95\% CI, 1.1-5.6]. Serious infections included one case each of urosepsis, staphylococcal cellulitis, anal abscess, sinusitis, appendicitis, Clostridium difficile infection, and herpes zoster [serious]. Four of these patients had prior infection events during OCTAVE Induction 1 and 2 or OCTAVE Sustain.

In the re-treatment subpopulation, six herpes zoster [non-serious and serious] events occurred in six patients, with an IR of 2.4 [95\% CI, 0.9-5.3] [Table 3]. Of the herpes zoster events, five were nonserious and all were resolved at data cut-off. One patient with herpes zoster [serious] permanently discontinued. There were no adjudicated opportunistic infections other than herpes zoster in the re-treatment subpopulation [herpes zoster opportunistic infection IR: 0.4 [95\% CI, 0.0-2.2]] [Table 3].

Non-melanoma skin cancer occurred in one patient, with an IR of 0.4 [95\% CI, 0.0-2.2]. There were three patients with malignancies in the re-treatment subpopulation, all of whom received a predominant dose of tofacitinib $10 \mathrm{mg}$ b.d. [average total daily dose $\geq 15 \mathrm{mg}$ ] during the tofacitinib UC clinical programme: hepatic angiosarcoma $[n=1]$ on Day 164 of OCTAVE Open, acute myeloid leukaemia $[n=1]$ on Day 310 of OCTAVE Open, and invasive ductal breast carcinoma $[n=1]$ on Day 642 of OCTAVE Open. The events of hepatic angiosarcoma and acute myeloid leukaemia resulted in death. The overall IR for death in the re-treatment population $(0.8$ [95\% CI, 0.12.8]) was similar to that in the overall cohort (0.2 [95\% CI, 0.1-0.6], respectively) [Table 3]. In the re-treatment subpopulation, one patient had a pulmonary embolism event on Day 174 of OCTAVE Open [236 days of tofacitinib exposure in total]. The patient had prior history of phlebothrombosis, stroke, hypertension, and hypercholesterolaemia. ${ }^{17}$ The event was resolved and did not result in the patient discontinuing tofacitinib therapy. One patient with prior history of hypertension had a major adverse cardiovascular event [stroke] on Day 786 of OCTAVE Open [859 days of tofacitinib exposure in total], which resulted in discontinuation of tofacitinib therapy. 


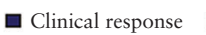

$\square$ Remission $\square$ Endoscopic improvement
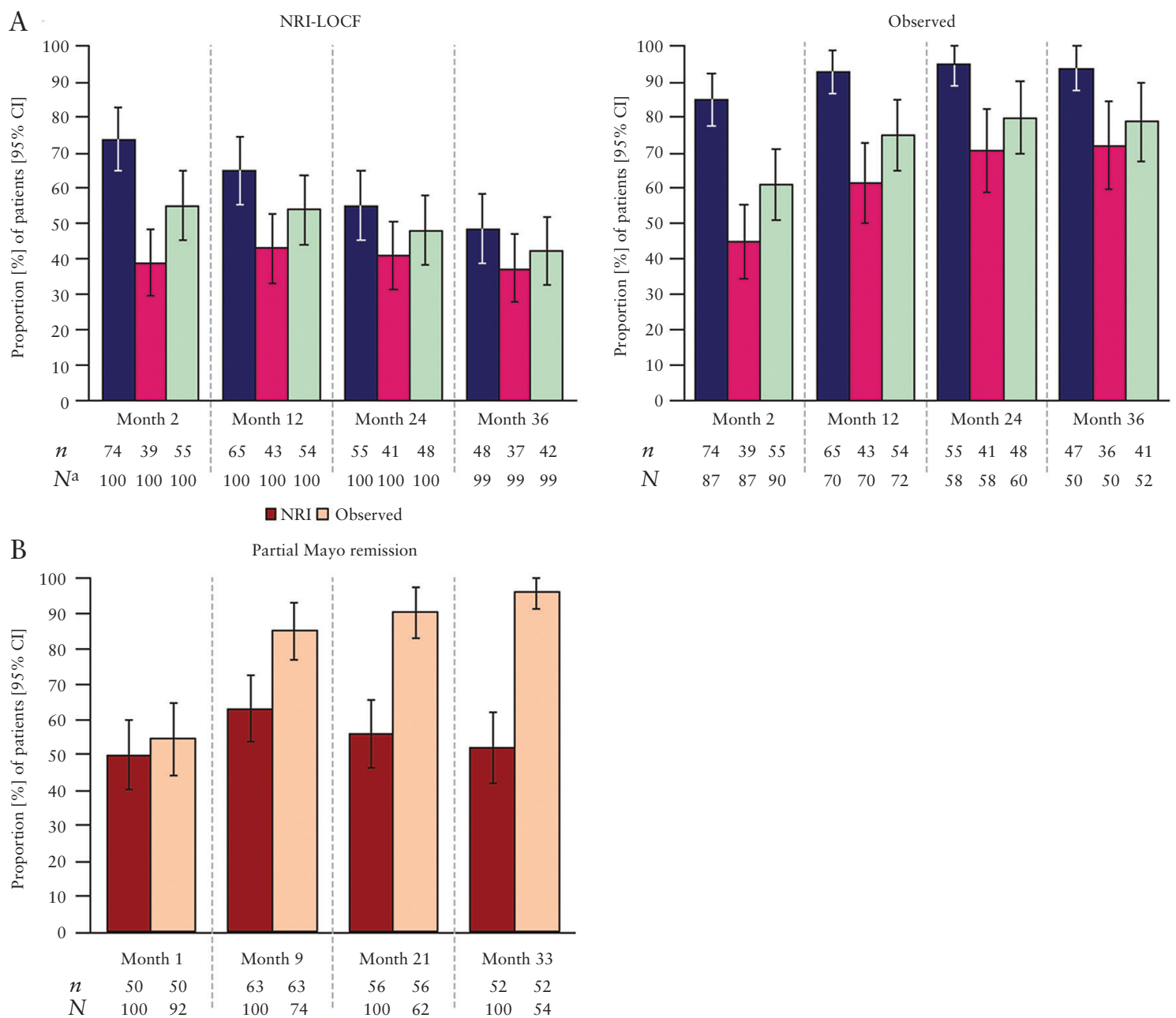

Figure 3. Proportion of patients in the tofacitinib re-treatment subpopulation who achieved [A] clinical response, remission, and endoscopic improvement at Months 2, 12, 24, and 36 of the OLE study [NRI-LOCF and observed data] and [B] partial Mayo remission at Months 1,9, 21, and 33 of the OLE study [NRI and observed data]. The re-treatment subpopulation comprised 100 patients who had clinical response at Week 8 with tofacitinib 10 mg b.d. in OCTAVE Induction 1 or 2, and subsequent treatment failure with placebo during OCTAVE Sustain. Per protocol, these patients received tofacitinib 10 mg b.d. in the OLE study. Induction remitters were patients with a total Mayo score $\leq 2$ and no subscore $>1$, and a rectal bleeding subscore of 0 at baseline of OCTAVE Sustain. Induction responders but non-remitters were patients who had clinical response but were not in remission at baseline of OCTAVE Sustain. Clinical response was defined as a decrease from induction study baseline total Mayo score of $\geq 3$ points and $30 \%$, plus a decrease in rectal bleeding subscore of $\geq 1$ point or an absolute rectal bleeding subscore of 0 or 1. Data from OCTAVE Open are presented up to May 27, 2019 [database not locked]. aLOCF at Month 36. b.d., twice daily; Cl, confidence interval; LOCF, last observation carried forward; $N$, number of evaluable patients with non-missing values; $n$, number of patients who achieved efficacy outcome; $\mathrm{NRI}$, non-responder imputation; OLE, open-label, long-term extension.

\section{Discussion}

This analysis assessed the efficacy and safety of tofacitinib $10 \mathrm{mg}$ b.d. in patients who had initial clinical response to tofacitinib $10 \mathrm{mg}$ b.d. as induction therapy, and subsequently re-initiated treatment with tofacitinib $10 \mathrm{mg}$ b.d. upon disease worsening following a period of treatment interruption of up to 52 weeks with placebo in OCTAVE Sustain. Median time to treatment failure was numerically higher in patients in remission at baseline of OCTAVE Sustain versus responders but non-remitters. Following tofacitinib re-treatment, recapture of efficacy occurred as early as Month 1, and could be observed up to Month 36. Tofacitinib $10 \mathrm{mg}$ b.d. can be re-initiated safely and efficaciously in the majority of patients with UC, regardless of prior TNFi failure.

Among tofacitinib induction responders, treatment interruption with placebo during OCTAVE Sustain led to relapse in the majority of patients within 52 weeks. However, patients in remission at baseline of OCTAVE Sustain maintained response for longer than responders but non-remitters [median time to treatment failure was 169 days versus 123 days, respectively]. This analysis provides an indication of how long it takes for patients to flare following treatment interruption, and further highlights the importance of maintenance therapy and the avoidance [where possible] of stopping treatment in a chronic inflammatory disease such as UC.

Interruption of treatment may be required for a variety of reasons, and this study has shown that for patients with prior response to tofacitinib $10 \mathrm{mg}$ b.d. induction therapy, re-treatment with tofacitinib $10 \mathrm{mg}$ b.d. appeared to be generally efficacious and well-tolerated in the majority of patients. As per the product label, the lowest effective dose required to maintain a response should be used; however, since patients in the re-treatment subpopulation 


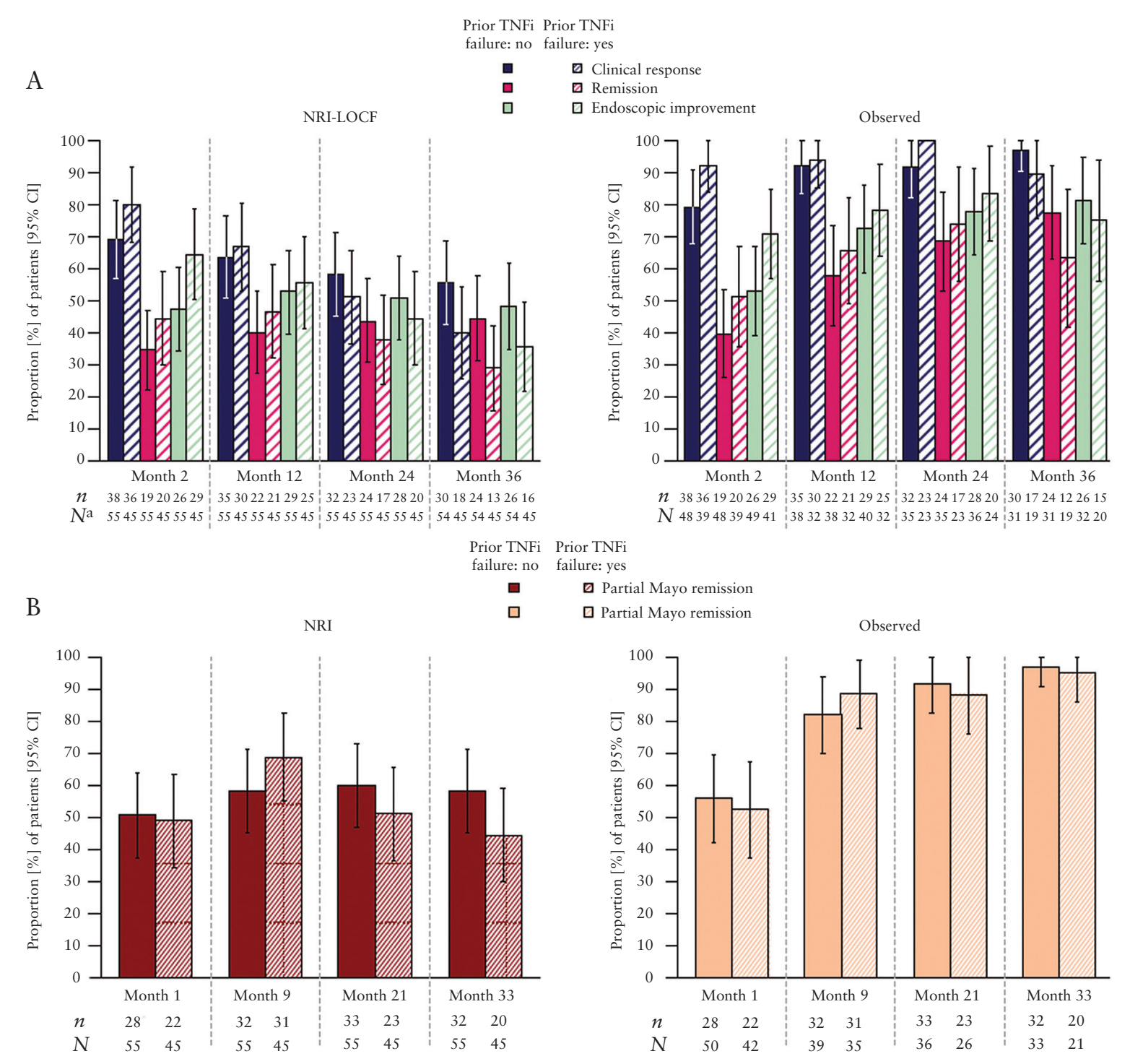

Figure 4. Proportion of patients in the tofacitinib re-treatment subpopulation who achieved [A] clinical response, remission, and endoscopic improvement at Months 2, 12, 24, and 36 of the OLE study [NRI-LOCF and observed data] and [B] partial Mayo remission at Months 1, 9, 21, and 33 of the OLE study, stratified by priorTNFi failure status [NRI and observed data]. The re-treatment subpopulation comprised 100 patients who had clinical response at Week 8 with tofacitinib $10 \mathrm{mg}$ b.d. in OCTAVE Induction 1 or 2 , and subsequent treatment failure with placebo during OCTAVE Sustain. Per protocol, these patients received tofacitinib $10 \mathrm{mg}$ b.d. in the OLE study. Induction remitters were patients with a total Mayo score $\leq 2$ and no subscore $>1$, and a rectal bleeding subscore of 0 at baseline of OCTAVE Sustain. Induction responders but non-remitters were patients who had clinical response but were not in remission at baseline of OCTAVE Sustain. Clinical response was defined as a decrease from induction study baseline total Mayo score of $\geq 3$ points and $30 \%$, plus a decrease in rectal bleeding subscore of $\geq 1$ point or an absolute rectal bleeding subscore of 0 or 1. Data from OCTAVE Open are presented up to May 27, 2019 [database not locked]. aLOCF at Month 36. b.d., twice daily; $\mathrm{Cl}$, confidence interval; LOCF, last observation carried forward; $N$, number of evaluable patients with non-missing values; $n$, number of patients who achieved efficacy outcome; NRI, non-responder imputation; OLE, open-label, long-term extension; TNFi, tumour necrosis factor inhibitor.

have relapsed following treatment interruption, re-treatment with the approved induction dose of $10 \mathrm{mg}$ b.d. can be considered. ${ }^{13,18}$ It should be noted that the tofacitinib $15 \mathrm{mg}$ b.d. induction dose was discontinued following a protocol amendment, and of the seven patients who received this induction dose, five patients experienced treatment failure while receiving placebo; these patients were excluded from the re-treatment subpopulation analysis. At Month 1 of re-treatment with tofacitinib $10 \mathrm{mg}$ b.d., $50.0 \%$ [NRI] of patients achieved partial Mayo remission and a decrease from baseline of OCTAVE Open in mean partial Mayo score [6.6 versus 2.4]. As partial Mayo remission does not include an endoscopic assessment, it represents a practical indicator of efficacy that can be measured more frequently than remission. Overall rates of clinical response, remission, and endoscopic improvement were $74.0 \%, 39.0 \%$, and $55.0 \%$ at Month 2, and $48.5 \%, 37.4 \%$, and $42.4 \%$ at Month 36 , respectively [NRI-LOCF]. Overall rates of partial Mayo remission were $50.0 \%$ at Month 1 and $52.0 \%$ at Month 33 [NRI].

The proportion of patients achieving efficacy outcomes was numerically greater in induction remitters versus induction responders but non-remitters. In contrast, prior TNFi failure status did not influence recapture of response; the proportions of patients achieving clinical and endoscopic outcomes were generally unaffected by prior TNFi failure status through to Month 24. This is likely related to the fact that this analysis selected patients who were already responders 

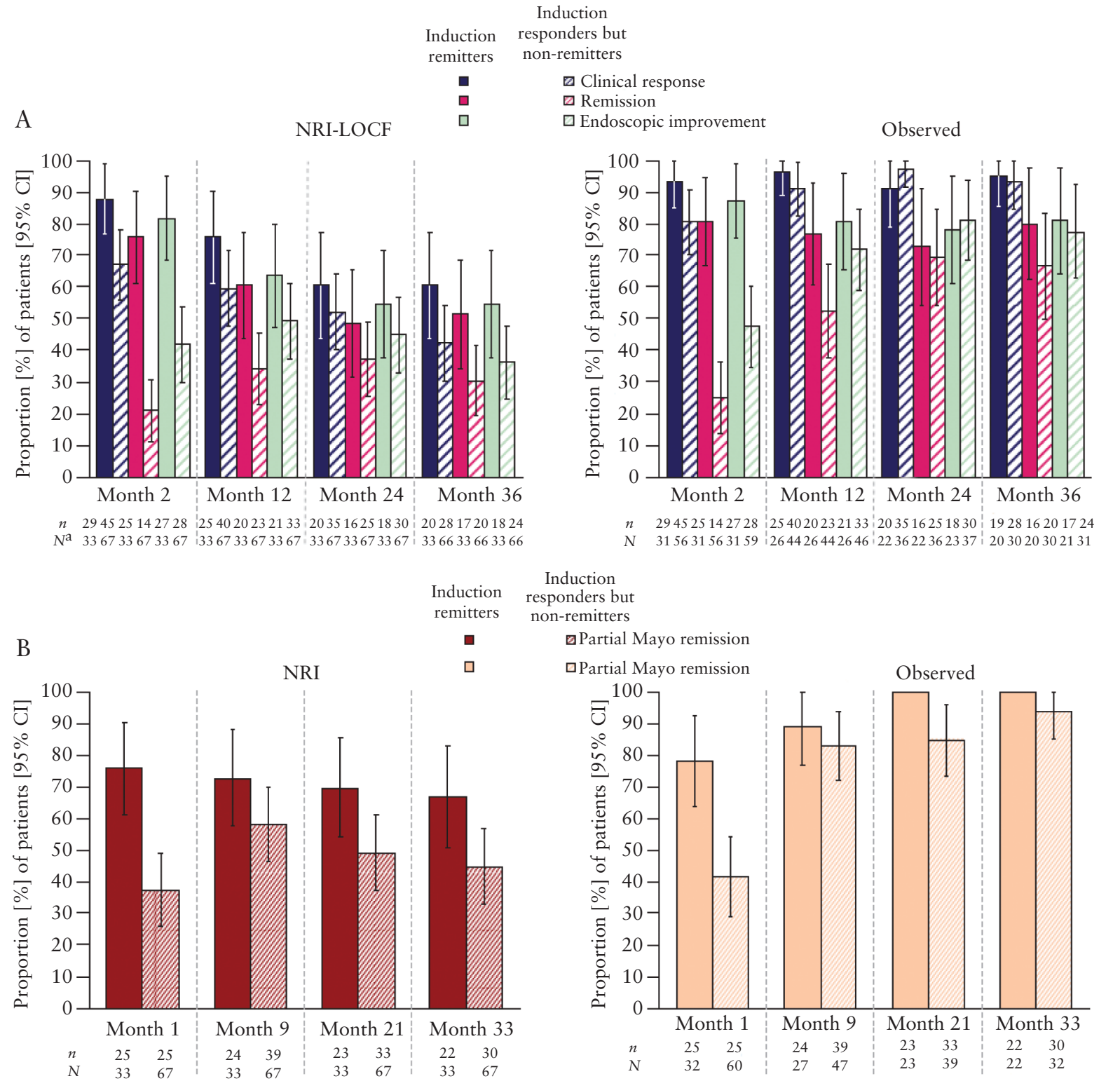

Figure 5. Proportion of patients in the tofacitinib re-treatment subpopulation who achieved [A] clinical response, remission, and endoscopic improvement at Months 2, 12, 24, and 36 of the OLE study [NRI-LOCF and observed data] and [B] partial Mayo remission at Months 1, 9, 21, and 33 of the OLE study, stratified by remission status at baseline of OCTAVE Sustain [NRI and observed data]. The re-treatment subpopulation comprised 100 patients who had clinical response at Week 8 with tofacitinib $10 \mathrm{mg}$ b.d. in OCTAVE Induction 1 or 2, and subsequent treatment failure with placebo during OCTAVE Sustain. Per protocol, these patients received tofacitinib $10 \mathrm{mg}$ b.d. in the OLE study. Induction remitters were patients with a total Mayo score $\leq 2$ and no subscore $>1$, and a rectal bleeding subscore of 0 at baseline of OCTAVE Sustain. Induction responders but non-remitters were patients who had clinical response but were not in remission at baseline of OCTAVE Sustain. Clinical response was defined as a decrease from induction study baseline total Mayo score of $\geq 3$ points and $30 \%$, plus a decrease in rectal bleeding subscore of $\geq 1$ point or an absolute rectal bleeding subscore of 0 or 1 . Data from OCTAVE Open are presented up to May 27,2019 [database not locked]. aLOCF at Month 36. b.d., twice daily; Cl, confidence interval; LOCF, last observation carried forward; $N$, number of evaluable patients with non-missing values; $n$, number of patients who achieved efficacy outcome; NRI, non-responder imputation; OLE, open-label, long-term extension.

to induction therapy. The proportions of patients achieving clinical response, remission, and endoscopic improvement at Month 36 were numerically greater in patients without prior TNFi failure versus patients with prior TNFi failure. While patients with prior failure to biologic therapy may represent a subgroup of patients with more longstanding [and therefore more refractory] disease, it is important to note the small sample size at these later time points.

In patients with UC treated with biologic therapies such as TNFi, the formation of neutralising anti-drug antibodies may reduce the efficacy of the biologic agent. ${ }^{5}$ Dose escalation or switching to an alternative TNFi may recapture response; however, the risk of lack of efficacy increases with successive TNFi agents. ${ }^{19,20}$ Furthermore, while dose-dependency of adverse events with TNFi therapies has not been demonstrated in patients with UC, ${ }^{21-23}$ concomitant treatment with immunomodulator therapy may be required to reduce immunogenicity and maintain efficacy. ${ }^{6}$ Immunomodulators are associated with additional safety concerns such as opportunistic infection $^{7}$ and cancer. ${ }^{8,9}$ As tofacitinib is a small molecule, it is not expected to induce the formation of neutralising anti-drug antibodies observed with TNFi agents. ${ }^{14}$ In these analyses, rates of 
Table 2. Significant predictors ${ }^{a}$ of recapture of clinical response in the tofacitinib re-treatment subpopulation in OCTAVE Open [NRI-LOCF].

\begin{tabular}{|c|c|c|c|c|}
\hline & \multicolumn{4}{|c|}{ Recapture of clinical response (odds ratio $[95 \% \mathrm{CI}]$ ) } \\
\hline & Month 2 & Month 12 & Month 24 & Month 36 \\
\hline Age [10-y increment $]$ & $1.59 *[1.06-2.38]$ & NS & NS & $1.54 *[1.09-2.17]$ \\
\hline $\begin{array}{l}\text { Mayo score at OLE study baseline [per 1-point } \\
\text { increase] }\end{array}$ & $0.61 *[0.40-0.91]$ & $0.69 *[0.51-0.93]$ & NS & NS \\
\hline Prior immunosuppressant failure [no vs yes] & NS & NS & NS & $3.51 *[1.21-10.15]$ \\
\hline $\begin{array}{l}\text { Oral corticosteroid use at Induction study baseline } \\
\text { [no vs yes] }\end{array}$ & $5.37 * *[1.72-16.72]$ & NS & $3.32 * *[1.45-7.59]$ & $2.61 *[1.09-6.24]$ \\
\hline
\end{tabular}

Odds ratios for association of significant predictors with recapture of clinical response are reported. The re-treatment subpopulation comprised 100 patients who had clinical response at Week 8 with tofacitinib $10 \mathrm{mg}$ b.d. in OCTAVE Induction 1 or 2, and subsequent treatment failure with placebo during OCTAVE Sustain. Per protocol, these patients received tofacitinib $10 \mathrm{mg}$ b.d. in the OLE study. Data from OCTAVE Open are presented up to May 27, 2019 [database not locked].

b.d., twice daily; BMI, body mass index; CI, confidence interval; LOCF, last observation carried forward; NRI, non-responder imputation; NS, not selected; OLE, open-label, long-term extension; y, year.

${ }^{2}$ Other non-significant predictors included in the stepwise logistic regression model were gender, weight $[\mathrm{kg}]$, height $[\mathrm{cm}]$, body mass index $[\mathrm{BMI}]\left[\mathrm{kg} / \mathrm{m}^{2}\right]$, prior TNFi failure [no vs yes], time since first diagnosis [y], remission at OCTAVE Sustain baseline [no vs yes], time to treatment failure [days], and oral corticosteroid use at OLE study baseline [no vs yes].

$* p<0.05 ; * *<<0.01$.

Table 3. Summary of safety and events of special interest in the tofacitinib re-treatment subpopulation of OCTAVE Open and in the overall cohort.

\begin{tabular}{|c|c|c|c|c|}
\hline & \multicolumn{2}{|c|}{$\begin{array}{l}\text { Tofacitinib re-treatment subpopulation } \\
{[N=100 ; 257.3 \mathrm{PY}]}\end{array}$} & \multicolumn{2}{|c|}{$\begin{array}{l}\text { Overall cohort; tofacitinib all } \\
{[N=1157 ; 2050.5 \mathrm{PY}]}\end{array}$} \\
\hline & $n[\%]$ & IR $[95 \% \mathrm{CI}]$ & $n[\%]$ & IR $[95 \% \mathrm{CI}]$ \\
\hline Serious adverse events & $25[25.0]$ & $10.4[6.7-15.4]$ & $189[16.3]$ & $9.5[8.2-11.0]$ \\
\hline Serious infection & $7[7.0]$ & $2.7[1.1-5.6]$ & $39[3.4]$ & $1.9[1.3-2.5]$ \\
\hline Opportunistic infection ${ }^{\mathrm{b}, \mathrm{d}}$ & $1[1.0]$ & $0.4[0.0-2.2]$ & $25[2.2]^{\mathrm{c}}$ & $1.2[0.8-1.8]^{\mathrm{c}}$ \\
\hline Herpes zoster [non-serious and serious] & $6[6.0]$ & $2.4[0.9-5.3]$ & $76[6.6]$ & $3.8[3.0-4.7]$ \\
\hline Malignancies [excluding NMSC],e & $3[3.0]$ & $1.2[0.2-3.4]$ & $13[1.2]^{\mathrm{c}}$ & $0.6[0.3-1.1]^{\mathrm{c}}$ \\
\hline $\mathrm{NMSC}^{\mathrm{b}, \mathrm{e}}$ & $1[1.0]$ & $0.4[0.0-2.2]$ & $16[1.4]^{\mathrm{c}}$ & $0.8[0.4-1.3]^{\mathrm{c}}$ \\
\hline $\mathrm{MACE}^{\mathrm{b}, \mathrm{e}}$ & $1[1.0]$ & $0.4[0.0-2.2]$ & $6[0.5]^{\mathrm{c}}$ & $0.3[0.1-0.6]^{\mathrm{c}}$ \\
\hline Deep vein thrombosis & $0[0.0]$ & $0.0[0.0-1.4]$ & $0[0.0]$ & $0.0[0.0-0.2]$ \\
\hline Pulmonary embolism & $1[1.0]$ & $0.4[0.0-2.2]$ & $4[0.3]$ & $0.2[0.1-0.5]$ \\
\hline Deaths $^{e}$ & $2[2.0]$ & $0.8[0.1-2.8]$ & $5[0.4]$ & $0.2[0.1-0.6]$ \\
\hline
\end{tabular}

Safety data from the re-treatment subpopulation are as of May 27, 2019 [database not locked]. The re-treatment subpopulation comprised patients who had clinical response at Week 8 with tofacitinib $10 \mathrm{mg}$ b.d. in OCTAVE Induction 1 or 2 , and subsequent treatment failure with placebo during OCTAVE Sustain. Per protocol, these patients received tofacitinib $10 \mathrm{mg}$ b.d. in the OLE study. IRs were calculated as the number of unique patients with events per $100 \mathrm{PY}$ of exposure. Events occurring within 28 days [except patients who were ongoing in OCTAVE Open at the time of this interim analysis] after the last dose are included for calculation of proportion and IR.

b.d., twice daily; CI, confidence interval; IR, incidence rate; MACE, major adverse cardiovascular events; $N$, number of patients treated in the treatment group; $n$, number of unique patients with one or more events; NMSC, non-melanoma skin cancer; OLE, open-label, long-term extension; PY, patient-years.

${ }^{a}$ Includes all patients receiving $\geq 1$ dose of tofacitinib 5 or $10 \mathrm{mg}$ b.d. in the phase 2 [NCT00787202] and phase 3 [OCTAVE Induction 1, NCT01465763; OCTAVE Induction 2, NCT01458951; OCTAVE Sustain, NCT01458574] studies and the OLE study [OCTAVE Open, NCT01470612], reported up to the November 2017 data cut-off. ${ }^{25}$

bAdjudicated events only.

${ }^{\mathrm{c}} \mathrm{N}=1124$ [excludes phase 2].

${ }^{\mathrm{d}}$ Excluding tuberculosis and herpes zoster with two adjacent dermatomes.

'Includes events that are outside the 28 day risk period.

recapture of efficacy responses among patients who had re-initiated tofacitinib therapy were generally high, suggesting that the loss of response during treatment interruption can be recaptured without switching therapies or mechanisms of action, and without adding concomitant agents.

It is important to note that not all patients in the re-treatment subpopulation recaptured efficacy responses, and while interruption of maintenance therapy is not generally recommended, identification of predictors of recapture of efficacy may enable physicians to identify patients in whom re-initiation of tofacitinib therapy is most likely to be successful. A stepwise logistic regression analysis was performed to identify potential factors associated with recapture of efficacy following re-treatment with tofacitinib. More severe disease at the time of re-treatment [as defined by total Mayo score] was associated with lower odds of recapture of clinical response, whereas increasing age, no prior immunosuppressant use, and no corticosteroid use at Induction study baseline were associated with recapture of clinical response. However, clinically relevant factors 
such as oral corticosteroid use at baseline of OCTAVE Open, prior TNFi failure, and remission status at baseline of OCTAVE Sustain were not significantly associated with recapture of clinical response up to Month 36 of tofacitinib re-treatment in this multivariable analysis. Therefore, although these regression analyses identified some factors associated with recapture of response, interruption of maintenance therapy is not generally recommended, and close monitoring of patients following treatment interruption and early re-initiation of therapy may be key to successful re-treatment.

Safety in the re-treatment subpopulation appeared to be consistent with that observed in the overall cohort in the tofacitinib UC clinical programme, although the sample size was small. No new safety risks were observed in this subpopulation of patients, compared with the previous safety update. ${ }^{24}$

A key limitation to consider for this analysis is the low number of patients in the re-treatment subpopulation. Based on the logistic regression analysis, it is challenging to predict patients in whom tofacitinib re-treatment would be successful, particularly at Months 24 and 36, due to patients discontinuing [including switching to other studies]. Larger studies based on real-world evidence should be conducted to further our understanding of the efficacy and safety of tofacitinib re-treatment following temporary interruption. Moreover, a valuable addition to this study would be to evaluate the optimal duration of re-treatment with tofacitinib $10 \mathrm{mg}$ b.d. before de-escalating to tofacitinib $5 \mathrm{mg}$ b.d.; this is a limitation of the current study, as patients who re-initiated treatment with tofacitinib $10 \mathrm{mg}$ b.d. continued on this dose.

These analyses suggest that although efficacy can be safely and successfully recaptured with tofacitinib $10 \mathrm{mg}$ b.d. re-treatment following treatment interruption in a substantial proportion of patients with UC, many patients failed to recapture efficacy. Therefore, although therapy with tofacitinib $10 \mathrm{mg}$ b.d. can be temporarily stopped to accommodate scenarios such as pregnancy, surgery, illness, adverse events, or a change in patient's insurance, treatment interruption without cause in patients with response to tofacitinib therapy is not generally recommended.

\section{Funding}

The clinical trials described in this article were sponsored by Pfizer Inc. Funding for medical writing support was provided by Pfizer Inc.

\section{Conflict of Interest}

JP has received personal fees from AbbVie, Arena, Boehringer Ingelheim, Celgene, Celltrion, Ferring, Galapagos, Genentech/Roche, GSK, Immunic, Janssen, Nestlé, Oppilan, Pfizer Inc, Progenity, Takeda, Theravance, and TiGenix. SV has received grant support from AbbVie, MSD, Pfizer Inc, and Takeda; speaker fees from AbbVie, Dr Falk Pharma, Ferring, Hospira, MSD, Takeda, and Tillotts; and consulting fees from AbbVie, Arena, Celgene, Eli Lilly, Ferring, Galapagos, Genentech/Roche, Gilead, Hospira, Janssen, MSD, Mundipharma, Pfizer Inc, Progenity, Second Genome, Shire, Takeda, and Theravance. MCD has received consulting fees from AbbVie, Bristol-Myers Squibb, Celgene, Gilead, Janssen, Pfizer Inc, Takeda, and UCB. EVL has received consulting fees from AbbVie, Allergan, Amgen, Arena, Boehringer Ingelheim, Bristol-Myers Squibb, Calibr, Celgene, Celltrion, Eli Lilly, Gilead, Genentech, Iterative Scopes, Janssen, Ono Pharma, Pfizer Inc, Sun Pharma, Takeda, and UCB; and grant support from AbbVie, Amgen, Bristol-Myers Squibb, Celgene, Genentech, Gilead, Janssen, Pfizer Inc, Receptos, Robarts Clinical Trials, Takeda, Theravance, and UCB. NL, WW, LS, CS, IM, and XG are employees and stockholders of Pfizer Inc. J-FC has received grant support from AbbVie, Janssen, and Takeda; payment for lectures from AbbVie, Allergan, Amgen, Ferring, Shire, and Takeda; consulting fees from AbbVie,
Amgen, Arena, Boehringer Ingelheim, Celgene, Celltrion, Eli Lilly, Enterome, Ferring, Geneva, Genentech, Immunic, Ipsen, Janssen, Landos, MedImmune, Merck, Novartis, O Mass, Otsuka, Pfizer Inc, Shire, Takeda, TiGenix, and Viela Bio; and holds stock options in Genfit and Intestinal Biotech Development.

\section{Author Contributions}

NL and CS planned the study/studies. NL and CS conducted the study/studies. JP, SV, MCD, EVL, NL, WW, LS, CS, IM, XG, and J-FC collected or interpreted data. JP, SV, MCD, EVL, NL, WW, LS, CS, IM, XG, and J-FC drafted and edited the manuscript. All authors approved the final version of the manuscript. Some of the data in the manuscript were previously presented at United European Gastroenterology Week Virtual 2020, October 11-13, 2020, and the American College of Gastroenterology Virtual Annual Scientific Meeting, October 23-28, 2020.

\section{Acknowledgements}

The authors would like to thank the patients, investigators, and study teams who were involved in the tofacitinib UC clinical programme: OCTAVE Induction 1, OCTAVE Induction 2, OCTAVE Sustain, and OCTAVE Open. These studies were sponsored by Pfizer Inc. Medical writing support, under the guidance of the authors, was provided by Sarah Mancini, PhD, CMC Connect, McCann Health Medical Communications and was funded by Pfizer Inc, New York, NY, USA in accordance with Good Publication Practice [GPP3] guidelines [Ann Intern Med 2015;163:461-4].

\section{Data Availability}

Upon request, and subject to review, Pfizer Inc. will provide the data that support the findings of this study. Subject to certain criteria, conditions, and exceptions, Pfizer Inc. may also provide access to the related individual anonymised participant data; see [https://www.pfizer.com/science/clinical-trials/ trial-data-and-results] for more information.

\section{Supplementary Data}

Supplementary data are available at ECCO-JCC online.

\section{References}

1. Ungaro R, Mehandru S, Allen PB, Peyrin-Biroulet L, Colombel JF. Ulcerative colitis. Lancet 2017;389:1756-70.

2. Danese S, Roda G, Peyrin-Biroulet L. Evolving therapeutic goals in ulcerative colitis: towards disease clearance. Nat Rev Gastroenterol Hepatol 2020;17:1-2.

3. Rubin DT. Restarting biologic agents after a drug holiday. Gastroenterol Hepatol (N Y) 2019;15:612-5.

4. Vermeire S, Gils A, Accossato P, Lula S, Marren A. Immunogenicity of biologics in inflammatory bowel disease. Therap Adv Gastroenterol 2018;11:1756283X17750355.

5. Vincent FB, Morand EF, Murphy K, Mackay F, Mariette X, Marcelli C. Antidrug antibodies [ADAb] to tumour necrosis factor [TNF]-specific neutralising agents in chronic inflammatory diseases: a real issue, a clinical perspective. Ann Rheum Dis 2013;72:165-78.

6. Baert F, Noman M, Vermeire S, et al. Influence of immunogenicity on the long-term efficacy of infliximab in Crohn's disease. N Engl J Med 2003;348:601-8.

7. Toruner M, Loftus EV Jr, Harmsen WS, et al. Risk factors for opportunistic infections in patients with inflammatory bowel disease. Gastroenterology 2008;134:929-36.

8. Annese V, Beaugerie L, Egan L, et al.; ECCO. European evidence-based consensus: inflammatory bowel disease and malignancies. J Crohns Colitis 2015;9:945-65.

9. Pasternak B, Svanström H, Schmiegelow K, Jess T, Hviid A. Use of azathioprine and the risk of cancer in inflammatory bowel disease. Am J Epidemiol 2013;177:1296-305. 
10. Sandborn WJ, Ghosh S, Panés J, et al.; Study A3921063 Investigators. Tofacitinib, an oral Janus kinase inhibitor, in active ulcerative colitis. $N$ Engl J Med 2012;367:616-24.

11. Sandborn WJ, Su C, Sands BE, et al.; OCTAVE Induction 1, OCTAVE Induction 2, and OCTAVE Sustain Investigators. Tofacitinib as induction and maintenance therapy for ulcerative colitis. N Engl J Med 2017;376:1723-36.

12. Lichtenstein GR, Loftus EV Jr, Wei SC, et al. Tofacitinib, an oral, smallmolecule Janus kinase inhibitor, in the treatment of ulcerative colitis: analysis of an open-label, long-term extension study with up to 5.9 years of treatment. J Crohns Colitis 2020;14:S100-1 [DOP61].

13. European Medicines Agency. Xelianz® [Tofacitinib] - Summary of Product Characteristics., 2020. https://www.ema.europa.eu/en/documents/product-information/xeljanz-epar-product-information_en.pdf Accessed June 12, 2020.

14. Mukherjee A, Hazra A, Smith MK, et al. Exposure-response characterization of tofacitinib efficacy in moderate to severe ulcerative colitis: results from a dose-ranging phase 2 trial. Br J Clin Pharmacol 2018;84:1136-45.

15. Dowty ME, Lin J, Ryder TF, et al. The pharmacokinetics, metabolism, and clearance mechanisms of tofacitinib, a Janus kinase inhibitor, in humans. Drug Metab Dispos 2014;42:759-73.

16. Sandborn WJ, Panés J, Panaccione R, et al. Tofacitinib for the treatment of ulcerative colitis: up to 5.4 years of safety data from global clinical trials. J Crohns Colitis 2019;13:S344 [P466].

17. Sandborn WJ, Panés J, Sands BE, et al. Venous thromboembolic events in the tofacitinib ulcerative colitis clinical development programme. Aliment Pharmacol Ther 2019;50:1068-76.
18. US Food and Drug Administration. Xeljanz® [Tofacitinib]: Highlights of Prescribing Information., 2019. https://labeling.pfizer.com/ShowLabeling. aspx?id=959 Accessed August 28, 2020.

19. Taxonera C, Rodríguez C, Bertoletti F, et al. [collaborators]. Clinical outcomes of golimumab as first, second or third anti-TNF agent in patients with moderate-to-severe ulcerative colitis. Inflamm Bowel Dis 2017;23:1394-402.

20. Olivera P, Danese S, Pouillon L, Bonovas S, Peyrin-Biroulet L. Effectiveness of golimumab in ulcerative colitis: a review of the real world evidence. Dig Liver Dis 2019;51:327-34.

21. Rutgeerts P, Sandborn WJ, Feagan BG, et al. Infliximab for induction and maintenance therapy for ulcerative colitis. $N$ Engl J Med 2005;353:2462-76.

22. Reinisch W, Sandborn WJ, Hommes DW, et al. Adalimumab for induction of clinical remission in moderately to severely active ulcerative colitis: results of a randomised controlled trial. Gut 2011;60:780-7.

23. Sandborn WJ, Feagan BG, Marano C, et al.; PURSUIT-SC Study Group. Subcutaneous golimumab induces clinical response and remission in patients with moderate-to-severe ulcerative colitis. Gastroenterology 2014;146:85-95.

24. Sandborn WJ, Panés J, D’Haens GR, et al. Safety of tofacitinib for treatment of ulcerative colitis, based on 4.4 years of data from global clinical trials. Clin Gastroenterol Hepatol 2019;17:1541-50.

25. Sandborn WJ, Panés J, Panaccione R, et al. Tofacitinib for the treatment of ulcerative colitis: up to 5.4 years of safety data from global clinical trials. Gastroenterology 2019;156:S1097 [Tu1717]. 